\title{
Existence and uniqueness of solutions for the Schrödinger integrable boundary value problem
}

\author{
Jianjie Wang ${ }^{1}$, Ali Mai ${ }^{2}$ and Hong Wang ${ }^{3 *}$
}

${ }^{\text {"Correspondence: }}$

wangh0629@tom.com

${ }^{3}$ Faculty of Science and Technology,

Universiti Kebangsaan Malaysia,

Bangi, Malaysia

Full list of author information is

available at the end of the article

\section{Abstract}

This paper is mainly devoted to the study of one kind of non differential equations. Under the integrable boundary alue cor ion, the existence and uniqueness of the solutions of this equation ar dis sed by using new Riesz representations of linear maps and the Schrödip r fixed $p$, rt theorem.

Keywords: Integrable boundary value; Nor ea - hrödinger differential equation; Schrödinger fixed point theorem

\section{Introduction}

The nonlinear Schrödinger reren 1 (NSD) equation is one of the most important inherently discrete mode's. NSD wations play a crucial role in the modeling of a great variety of phenomen ar ing from solid state and condensed matter physics to biology [1-4]. For examr ie, they seen successfully applied to the modeling of localized pulse propagation ptl fibers and wave guides, to the study of energy relaxation in solids, to the behavior of amor . ous material, to the modeling of self-trapping of vibrational energy in prot ns or studies related to the denaturation of the NSD double strand [5].

In 196 Gross considered a NSD equation with Dirac distribution defect (see [6]),

$$
\left\langle u_{t}\right)^{\frac{1}{2}} u_{x x}+q \delta_{a} u+g\left(|u|^{2}\right) u=0 \quad \text { in } \boldsymbol{\Omega} \times \mathbb{R}_{+} \text {, }
$$

ere $\boldsymbol{\Omega} \subset \mathbb{R}, u=u(x, t)$ is the unknown solution maps $\boldsymbol{\Omega} \times \mathbb{R}_{+}$into $\mathbb{C}$, $\delta_{a}$ is the Dirac distribution at the point $a \in \boldsymbol{\Omega}$, namely, $\left\langle\delta_{a}, v\right\rangle=v(a)$ for $v \in \mathbf{H}^{1}(\boldsymbol{\Omega})$, and $q \in \mathbb{R}$ represents its intensity parameter. Such a distribution is introduced in order to model physically the defect at the point $x=a$ (see [7]). The function g represents a generalization of the classical nonlinear Schrödinger equation (see for example [8]). As for other contributions to the analysis of nonlinear Schrödinger equations, we refer to Refs. [9-12] and the references therein.

In this paper, we consider the following NSD equation:

$$
\mathfrak{X}_{s}=x+\int_{0}^{s} b\left(s, \mathfrak{X}_{s}\right) d s+\int_{0}^{s} h\left(s, \mathfrak{X}_{s}\right) d\langle\mathfrak{B}\rangle_{s}+\int_{0}^{s} \sigma\left(s, \mathfrak{X}_{s}\right) d \mathfrak{B}_{s}
$$

where $0 \leq s \leq S$ and $\langle\mathfrak{B}\rangle$ is the quadratic variation of the Brownian motion $\mathfrak{B}$.

(c) The Author(s) 2018. This article is distributed under the terms of the Creative Commons Attribution 4.0 International License (http://creativecommons.org/licenses/by/4.0/), which permits unrestricted use, distribution, and reproduction in any medium, provided you give appropriate credit to the original author(s) and the source, provide a link to the Creative Commons license, and indicate if changes were made. 
It is worth mentioning that (1) comes from an expansion of the Feynman path integral from Brownian-like to Lévy-like quantum mechanical paths (see [13] for details). When the coefficients $b, h$ and $\sigma$ are constants in (1), the Lévy dynamics becomes the Brownian dynamics, and (1) reduces to the classical stochastic differential equation

$$
\mathfrak{Y}_{s}=\xi+\int_{s}^{S} f\left(s, \mathfrak{Y}_{s}, \mathfrak{Z}_{s}\right) d s+\int_{s}^{S} g\left(s, \mathfrak{Y}_{s}, \mathfrak{Z}_{s}\right) d\langle B\rangle_{s}-\int_{s}^{S} \mathfrak{Z}_{s} d \mathfrak{B}_{s}-\left(\mathfrak{K}_{S}-\mathfrak{K}_{s}\right)
$$

under standard Lipschitz conditions on $f(s, y, z), g(s, y, z)$ in $y, z$ and the $L_{G}^{p}\left(\Omega_{S}\right)(p>$ integrability condition on $\xi$. The solution $(\mathfrak{Y}, \mathfrak{Z}, \mathfrak{K})$ is universally defined in the sp $-\mathfrak{z}$ of the Schrödinger framework, in which the processes have a strong regularity pro serty. It should be noted that $K$ is a decreasing Schrödinger martingale.

It is well known that classical stochastic differential equations are enrou. red wren one applies the stochastic maximum principle to optimal stochastic ontrol p. Jlems. Such equations are also encountered in the probabilistic interpretation general type of systems quasilinear PDEs, as well as in finance (see [13-15] for tails).

The rest of this paper is organized as follows. In Sect. 2, we to sme notions and results. In Sect. 3, the main results and their proofs are presentec

\section{Preliminaries}

In this section, we introduce some notation a prelin hnary results in Schrödinger framework which are needed in the follow sect . More details can be found in [1619].

Let $\Gamma_{S}=C_{0}([0, S] ; R)$, the space of $\quad 1 \mathrm{v}$, lued continuous functions on $[0, S]$ with $w_{0}=0$, be endowed with the distance ( $-\mathrm{e}[20]$,

$$
\left.d\left(w^{1}, w^{2}\right):=\sum_{N=1}^{\infty} \frac{\left(\max <_{s \leq N} \mid w_{s}\right.}{2^{N}}\right) \frac{\left.w_{s}^{2} \mid\right) \wedge 1}{2}
$$

and let $\mathfrak{B}_{s}(w)=w$ be the unical process. Denote by $\mathbb{F}:=\left\{\mathcal{F}_{s}\right\}_{0 \leq s \leq S}$ the natural filtration generated by $\left.\mathfrak{B}_{s}, \mathfrak{t}_{2}, \Gamma_{s}\right)$ be the space of all $\mathbb{F}$-measurable real functions. Let

$$
\left.L \Gamma_{S}\right)-\left\{\phi\left(\mathfrak{S}_{s_{1}}, \ldots, \mathfrak{B}_{s_{n}}\right): \forall n \geq 1, s_{1}, \ldots, s_{n} \in[0, S], \forall \phi \in C_{b, L_{i p}}\left(R^{n}\right)\right\},
$$

re $C_{b, L_{i p}} R^{n}$ ) denotes the set of bounded Lipschitz functions in $R^{n}$ (see [21]).

In sequel, we will work under the following assumptions.

(H1) For $u \in R^{3}, \varepsilon>0, \Phi(x) \in L_{G}^{2}\left(\Gamma_{S}\right), f(\cdot, u), g(\cdot, u), b(\cdot, u), h(\cdot, u), \sigma(\cdot, u) \in M_{G}^{2}(0, S)$;

(H2) For $u^{1}, u^{2} \in R^{3}$, there exists a positive constant $C_{1}$ such that

$$
\left\|f\left(s, u^{1}\right)-f\left(s, u^{2}\right)\right\| \vee\left\|b\left(s, u^{1}\right)-f\left(s, u^{2}\right)\right\| \vee\left\|A\left(s, u^{1}\right)-A\left(s, u^{2}\right)\right\| \leq C_{1}\left\|u^{1}-u^{2}\right\|
$$

and

$$
\left\|\Phi\left(x^{1}\right)-\Phi\left(x^{2}\right)\right\| \leq C_{1}\left\|x^{1}-x^{2}\right\| ;
$$

(H3) For $u^{1}, u^{2} \in R^{3}$, there exists a positive constant $C_{2}$ such that

$$
\left[A\left(s, u^{1}\right)-A\left(s, u^{2}\right), u^{1}-u^{2}\right] \leq-C_{2}\left\|u^{1}-u^{2}\right\|^{2} .
$$


A sublinear functional on $L_{i p}\left(\Gamma_{S}\right)$ satisfies: for all $\mathfrak{X}, \mathfrak{Y} \in L_{i p}\left(\Gamma_{S}\right)$,

(I) monotonicity: $\mathfrak{E}[\mathfrak{X}] \geq \mathfrak{E}[\mathfrak{Y}]$ if $\mathfrak{X} \leq \mathfrak{Y}$;

(II) constant preserving: $\mathfrak{E}[C]=C$ for $C \in R$;

(III) sub-additivity: $\mathfrak{E}[\mathfrak{X}+\mathfrak{Y}] \leq \mathfrak{E}[\mathfrak{X}]+\mathfrak{E}[\mathfrak{Y}]$;

(IV) positive homogeneity: $\mathfrak{E}[\lambda \mathfrak{X}]=\lambda \mathfrak{E}[\mathfrak{X}]$ for $\lambda \geq 0$.

The tripe $\left(\Gamma, L_{i p}\left(\Gamma_{S}\right), \mathfrak{E}\right)$ is called a sublinear expectation space and $E$ is called a sublinear expectation.

Definition 2.1 (see [22]) A random variable $\mathfrak{X} \in L_{i p}\left(\Gamma_{S}\right)$ is the Schrödinger normal dis tributed with parameters $\left(0,\left[\underline{\sigma}^{2}, \bar{\sigma}^{2}\right]\right)$, i.e., $\mathfrak{X} \sim N\left(0,\left[\underline{\sigma}^{2}, \bar{\sigma}^{2}\right]\right)$ if for each $\phi \in C_{b, L_{i p}}($ R),

$$
u(s, x):=\mathfrak{E}[\phi(x+\sqrt{t} \mathfrak{X})]
$$

is a viscosity solution to the following PDE:

$$
\left\{\begin{array}{l}
\frac{\partial u}{\partial s}+G \frac{\partial^{2} u}{\partial x^{2}}=0 \\
u_{s_{0}}=\phi(x),
\end{array}\right.
$$

on $R^{+} \times R$, where

$$
G(a):=\frac{a^{+} \bar{\sigma}^{2}-a^{-} \underline{\sigma}^{2}}{2}
$$

and $a \in R$.

Definition 2.2 (see [23]) We cal sublinear expectation $\hat{\mathfrak{E}}: L_{i p}\left(\Gamma_{S}\right) \rightarrow R$ a Schrödinger expectation if the cano ical process $\mathcal{B}$ is a Schrödinger Brownian motion under $\hat{\mathfrak{E}}[\cdot]$, that is, for each $0 \leq s \leq t \leq S$, the ir crement $\mathfrak{B}_{s}-\mathfrak{B}_{s} \sim N\left(0,\left[\underline{\sigma}^{2}(s-s), \bar{\sigma}^{2}\right]\right)(s-s)$ and for all $n>0,0 \leq s_{1} \leq \cdots \leq s_{n} \leq \quad i \varphi \in L_{i p}\left(\Gamma_{S}\right)$

$$
\left.\hat{\mathfrak{E}}\left[\varphi^{\prime}, \ldots, \vartheta_{s_{n-1}}, \mathfrak{B}_{s_{n}}-\mathfrak{B}_{s_{n-1}}\right)\right]=\hat{\mathfrak{E}}\left[\psi\left(\mathfrak{B}_{s_{1}}, \ldots, \mathfrak{B}_{s_{n-1}}\right)\right] \text {, }
$$

$\mathrm{w}^{\prime}$ iere

$$
\left.{ }_{4}, \ldots, x_{n-1}\right):=\hat{\mathfrak{E}}\left[\varphi\left(x_{1}, \ldots, x_{n-1}, \sqrt{s_{n}-s_{n-1}} \mathfrak{B}_{1}\right)\right]
$$

We can also define the conditional Schrödinger expectation $\hat{\mathfrak{E}}_{s}$ of $\xi \in L_{i p}\left(\Gamma_{S}\right)$ knowing $L_{i p}(\Gamma t)$ for $t \in[0, S]$. Without loss of generality, we can assume that $\xi$ has the representation

$$
\xi=\varphi\left(\mathfrak{B}\left(s_{1}\right), \mathfrak{B}\left(s_{2}\right)-\mathfrak{B}\left(s_{1}\right), \ldots, \mathfrak{B}\left(s_{n}\right)-\mathfrak{B}\left(s_{n_{1}}\right)\right)
$$

with $t=s_{i}$, for some $1 \leq i \leq n$, and we put

$$
\begin{gathered}
\hat{\mathfrak{E}}_{s_{i}}\left[\varphi\left(\mathfrak{B}\left(s_{1}\right), \mathfrak{B}\left(s_{2}\right)-\mathfrak{B}\left(s_{1}\right), \ldots, \mathfrak{B}\left(s_{n}\right)-\mathfrak{B}\left(s_{n-1}\right)\right)\right] \\
=\tilde{\varphi}\left(\mathfrak{B}\left(s_{1}\right), \mathfrak{B}\left(s_{2}\right)-\mathfrak{B}\left(s_{1}\right), \ldots, \mathfrak{B}\left(s_{i}\right)-\mathfrak{B}\left(s_{i-1}\right)\right),
\end{gathered}
$$


where

$$
\tilde{\varphi}\left(x_{1}, \ldots, x_{i}\right)=\hat{\mathfrak{E}}\left[\varphi\left(x_{1}, \ldots, x_{i}, \mathfrak{B}\left(s_{i+1}\right)-\mathfrak{B}\left(s_{i}\right), \ldots, \mathfrak{B}\left(s_{n}\right)-\mathfrak{B}\left(s_{n-1}\right)\right)\right]
$$

For $p \geq 1$, we denote by $L_{G}^{p}\left(\Gamma_{S}\right)$ the completion of $L_{i p}\left(\Gamma_{S}\right)$ under the natural norm

$$
\|\mathfrak{X}\|_{p, G}:=\left(\hat{\mathfrak{E}}\left[|\mathfrak{X}|^{p}\right]\right)^{\frac{1}{p}} .
$$

$\hat{\mathfrak{E}}$ is a continuous mapping on $L_{i p}\left(\Gamma_{S}\right)$ endowed with the norm $\|\cdot\|_{1, G}$. Therefore, it can $\mathrm{D}$ extended continuously to $L_{G}^{1}\left(\Gamma_{S}\right)$ under the norm $\|X\|_{1, G}$.

Next, we introduce the Itô integral of Schrödinger Brownian motion.

Let $M_{G}^{0}(0, S)$ be the collection of processes in the following form: for a $g$. $\mathrm{p}$ $\pi_{S}=\left\{s_{0}, s_{1}, \ldots, s_{N}\right\}$ of $[0, S]$, set

$$
\eta_{s}(w)=\sum_{k=0}^{N-1} \xi_{k}(w) I_{\left[s_{k}, s_{k+1}\right)}(s),
$$

where $\xi_{k} \in L_{i p}\left(\Gamma_{t k}\right)$ and $k=0,1, \ldots, N-1$ are given.

For $p \geq 1$, we denote by $H_{G}^{p}(0, S), M_{G}^{p}(0, S)$ the complet orr ${ }_{G}^{9}(0, S)$ under the norm

$$
\|\eta\|_{H_{G}^{p}(0, S)}=\left\{\hat{\mathfrak{E}}\left[\left(\int_{0}^{S}\left|\eta_{s}\right|^{2} d s\right)^{\frac{p}{2}}\right]\right\}^{\frac{1}{p}}
$$

and

$$
\|\eta\|_{M_{G}^{p}(0, S)}=\left\{\hat{\mathfrak{E}}\left[\left(\left.\int_{0}^{S}\right|_{1,||^{p} a}\right\rangle\right\}^{\frac{1}{p}},\right.
$$

respectively. It is easy see tha)

$$
H_{G}^{2}(0, S)=\lambda_{G} \mathrm{C}
$$

As ir \{24], or eac $\measuredangle \eta \in H_{G}^{p}(0, S)$ with $p \geq 1$, we can define Itô integral $\int_{0}^{S} \eta_{s} d \mathfrak{B}_{s}$. Moreove, the lowng $B-D-G$ inequality holds.

t $\mathfrak{G}_{G}^{\alpha}(0, j)$ denote the collection of processes $(\mathfrak{Y}, \mathfrak{Z}, \mathfrak{K})$ such that $\mathfrak{Y} \in S_{G}^{\alpha}(0, S), \mathfrak{Z} \in$ $H_{G}^{\alpha}(\checkmark\urcorner, K$ is a decreasing Schrödinger martingale with $\mathfrak{K}_{0}=0$ and $\mathfrak{K}_{S} \in L_{G}^{\alpha}(\Gamma)$.

Le nma 2.1 (see [25]) Assume that $\xi \in L_{G}^{\beta}\left(\Gamma_{S}\right), f, g \in M_{G}^{\beta}(0, S)$ and satisfy the Lipschitz condition for some $\beta>1$. Then Eq. (2) has a unique solution $(\mathfrak{Y}, \mathfrak{Z}, \mathfrak{K}) \in \mathfrak{G}_{G}^{\alpha}(0, S)$ for any $1<\alpha<\beta$.

In [26], the authors also got the explicit solution of the following special type of NSD equation.

Lemma 2.2 Assume that $\left\{a_{s}\right\}_{s \in[0, S]},\left\{c_{s}\right\}_{s \in[0, S]}$ are bounded processes in $M_{G}^{1}(0, S)$ and $\xi \in$ $L_{G}^{1}\left(\Gamma_{S}\right),\left\{m_{s}\right\}_{s \in[0, S]},\left\{n_{s}\right\}_{s \in[0, S]} \in M_{G}^{1}(0, S)$. Then the NSD equation

$$
\mathfrak{Y}_{s}=\hat{\mathfrak{E}}_{s}\left[\xi+\int_{s}^{S}\left(a_{s} \mathfrak{Y}_{s}+m_{s}\right) d s+\int_{s}^{S}\left(c_{s} \mathfrak{Y}_{s}+n_{s}\right) d\langle\mathfrak{B}\rangle_{s}\right]
$$


has an explicit solution,

$$
\mathfrak{Y}_{s}=\left(\mathfrak{X}_{s}\right)^{-1} \hat{\mathfrak{E}}_{s}\left[\mathfrak{X}_{S} \xi+\int_{s}^{S}\left(m_{s}\right) d s+\int_{s}^{S}\left(n_{s}\right) d\langle\mathfrak{B}\rangle_{s}\right]
$$

where

$$
\mathfrak{X}_{s}=\exp \left(\int_{0}^{s} a_{s} d s+\int_{0}^{s} c_{s} d\langle\mathfrak{B}\rangle_{s}\right)
$$

Lemma 2.3 (see [27]) Suppose that a nonnegative real sequence $\left\{a_{i}\right\}_{i=1}^{\infty}=1$ satisfy.ng

$$
8 a_{i+1} \leq 2 a_{i}+a_{i-1}
$$

for any $i \geq 1$. Then there exists a positive constant $c$, such that $2^{i} a_{i} \leq c J_{c}$, ny $i \geq j$.

\section{Main results and their proofs}

In this section, we introduce the main results and their proofs.

Let $u:=(x, y, z), A(s, u):=(-g(s, u), h(s, u), \sigma(s, u))$. [., .] motes th.e usual inner product in real number space and $|\cdot|$ denotes the Euclidean norm

Our first main result can be summarized as follows.

Theorem 3.1 Suppose that $(\mathrm{H} 1)-(\mathrm{H} 3)$ are si fied Then there exists $s \in[0, S]$ such that (1) has a nontrivial and nonnegative olution.

Proof Let a nonnegative real uence $\{, y\}\}_{k \in \mathbb{N}} \subset \mathbb{F}$ such that $\left\{A\left(s, u^{(k)}\right)\right\}_{k \in \mathbb{N}}$ is bounded Lipschitz functions in $R^{n}$ ana

$$
\lim _{k \rightarrow \infty}\left(1+\left\|u^{(k)}\right\|\right)\left\|,\left(s, u^{(k)}\right)\right\|=0 .
$$

So there exists a pe constant $C_{3}$ such that $\left|A\left(s, u^{(k)}\right)\right| \leq C_{3}$ (see [28]), which concludes th.au

$$
\begin{aligned}
2 C_{3} & =? A\left(s, u^{(k)}\right)-\left\langle A^{\prime}\left(s, u^{(k)}\right), u^{(k)}\right\rangle \\
y & =\sum_{n=-\infty}^{+\infty} \gamma_{n}\left[g\left(s, u_{n}^{(k)}\right) u_{n}^{(k)}-2 h\left(s, u_{n}^{(k)}\right)\right] .
\end{aligned}
$$

It follows from (H1) and (4) that

$$
\left|F\left(u_{n}\right)\right| \leq \frac{v-\omega}{4 \bar{\gamma}} u_{n}^{2}
$$

for any $\left|u_{n}\right| \leq \eta$, where $n \in \mathbb{Z}$ and $\eta$ is a positive real number satisfying $\eta \in(0,1)$.

Then (H2) and (5) immediately give

$$
\begin{aligned}
& g\left(s, u_{n}^{(k)}\right) u_{n}^{(k)}>2 h\left(s, u_{n}^{(k)}\right) \geq 0, \\
& h\left(s, u_{n}^{(k)}\right) \leq\left[p+q\left|u_{n}^{(k)}\right|^{\mu / 2}\right]\left[g\left(s, u_{n}^{(k)}\right) u_{n}^{(k)}-2 h\left(s, u_{n}^{(k)}\right)\right] .
\end{aligned}
$$


By Lemma 2.3, (6) and (7), we have

$$
\begin{aligned}
& \frac{1}{2}\left\|u^{(k)}\right\|^{2} \\
& =A\left(s, u^{(k)}\right)+\frac{\tau}{2}\left\|u^{(k)}\right\|_{l^{2}}^{2}+\sum_{n \in \mathbb{Z}\left(\left|u_{n}^{(k)}\right| \leq \eta\right)} \varrho_{n} h\left(s, u_{n}^{(k)}\right)+\sum_{n \in \mathbb{Z}\left(\left|u_{n}^{(k)}\right| \geq \eta\right)} \varrho_{n} h\left(s, u_{n}^{(k)}\right) \\
& \leq A\left(s, u^{(k)}\right)+\frac{\tau}{2 \underline{\underline{v}}}\left\|u^{(k)}\right\|^{2}+\frac{\underline{v}-\tau}{4} \sum_{n \in \mathbb{Z}\left(\left|u_{n}^{(k)}\right| \leq \eta\right)}\left(u_{n}^{(k)}\right)^{2} \\
& \quad+\bar{\varrho} \sum_{n \in \mathbb{Z}\left(\left|u_{n}^{(k)}\right| \geq \eta\right)}\left[p+q\left|u_{n}^{(k)}\right|^{\mu / 2}\right]\left[g\left(s, u_{n}^{(k)}\right) u_{n}^{(k)}-2 h\left(s, u_{n}^{(k)}\right)\right] \\
& \leq c+\frac{\tau}{2 \underline{v}}\left\|u^{(k)}\right\|^{2}+\frac{\underline{v}-\tau}{4 \underline{v}}\left\|u^{(k)}\right\|^{2}+2 c \bar{\varrho}\left(p+q \underline{v}^{\mu / 2}\left\|u^{(k)}\right\|^{\mu}\right),
\end{aligned}
$$

which gives

$$
\frac{\underline{v}-\tau}{4 \underline{v}}\left\|u^{(k)}\right\|^{2} \leq c+2 c \bar{\varrho}\left(p+q \underline{v}^{\mu / 2}\left\|u^{(k)}\right\|^{\mu}\right) .
$$

It is obvious that the nonnegative real sequence $\left\{u^{(k)}\right\}_{k \in}$ a positive constant $C_{4}$ such that (see [29])

$$
\left\|u^{(k)}\right\| \leq C_{4}
$$

for any $k \in \mathbb{N}$, which gives $u^{(k)} \rightarrow u^{(0)}$ n $\Sigma$ as $k \rightarrow \infty$.

Let $\varepsilon$ be a given number. Then unere ists positive number $\zeta$ such that

$$
|g(s, u)| \leq \varepsilon|u|
$$

for any $u \in \mathbb{R}$ from $(\mathrm{H}$ where $|u| \leq \zeta$.

It follows from $(\mathrm{H} 1) \mathrm{t}_{1} \quad$ re exists a positive integer $C_{5}$ satisfying

$$
\zeta^{2} v_{n}>C_{5}^{2}
$$

for ny,

$$
\text { v (8), (9, 1d (10), we obtain }
$$

$$
\varphi_{,}\left(u_{n}^{(k)}\right)^{2}=C_{5}^{2} v_{n}\left(u_{n}^{(k)}\right)^{2} \leq v_{n} \zeta^{2}\left\|u^{(k)}\right\|^{2} \leq C_{5}^{2} v_{n} \zeta^{2}
$$

for any $|n| \geq C_{5}$.

Since $u^{(k)} \rightarrow u^{(0)}$ in $E$ as $k \rightarrow \infty$, it is obvious that $u_{n}^{(k)}$ converges to $u_{n}^{(0)}$ pointwise for all $n \in \mathbb{Z}$, that is,

$$
\lim _{k \rightarrow \infty} u_{n}^{(k)}=u_{n}^{(0)}
$$

for any $n \in \mathbb{Z}$, which together with (11) gives

$$
\left(u_{n}^{(0)}\right)^{2} \leq \zeta^{2}
$$

for any $|n| \geq C_{5}$. 
It follows from (12), (13) and the continuity of $g(s, u)$ on $u$ that there exists a positive integer $C_{6}$ such that

$$
\sum_{n=-D}^{D} \varrho_{n}\left|f\left(u_{n}^{(k)}\right)-f\left(u_{n}^{(0)}\right)\right|<\varepsilon
$$

for any $k \geq C_{6}$.

Meanwhile, we have

$$
\begin{aligned}
& \sum_{|n| \geq D} \varrho_{n}\left|f\left(u_{n}^{(k)}\right)-g\left(s, u_{n}^{(0)}\right)\right|\left|u_{n}^{(k)}-u_{n}^{(0)}\right| \\
& \leq \sum_{|n| \geq D} \bar{\varrho}\left(\left|f\left(u_{n}^{(k)}\right)\right|+\left|g\left(s, u_{n}^{(0)}\right)\right|\right)\left(\left|u_{n}^{(k)}\right|+\left|u_{n}^{(0)}\right|\right) \\
& \leq \bar{\varrho} \varepsilon \sum_{|n| \geq D}\left[\left|u_{n}^{(k)}\right|+\left|u_{n}^{(0)}\right|\right]\left(\left|u_{n}^{(k)}\right|+\left|u_{n}^{(0)}\right|\right) \\
& \leq 2 \bar{\varrho} \varepsilon \sum_{n=-\infty}^{+\infty}\left(\left|u_{n}^{(k)}\right|^{2}+\left|u_{n}^{(0)}\right|^{2}\right) \\
& \leq \frac{2 \varrho \varepsilon}{\underline{v}}\left(K_{1}^{2}+\left\|u^{(0)}\right\|^{2}\right)
\end{aligned}
$$

from (H3), (8), (9) and the Hölder inequali:

Since $\varepsilon$ is arbitrary, we obtain

$$
\sum_{n=-\infty}^{+\infty} \varrho_{n} \mid g\left(s, u_{n}^{(k)}\right)-g\left(s, u_{\eta}^{(0)} \rightarrow 0\right.
$$

as $k \rightarrow \infty$.

It follows that

$$
\begin{aligned}
& \left.\left\langle A^{\prime}\left(s, u^{(k)}\right)-u^{(0)}\right), u^{(k)}-u^{(0)}\right\rangle \\
& \geqslant \frac{v}{\underline{v}}\left\|u^{(k)}-u^{(0)}\right\|^{2}-\sum_{n=-\infty}^{+\infty} \varrho_{n}\left(g\left(s, u_{n}^{(k)}\right)-g\left(s, u_{n}^{(0)}\right)\right)\left(u^{(k)}-u^{(0)}\right)
\end{aligned}
$$

from (14), (15) and (16), which gives

$$
\begin{aligned}
\frac{\underline{\nu}-\tau}{\underline{v}}\left\|u^{(k)}-u^{(0)}\right\|^{2} \leq & \left\langle A^{\prime}\left(s, u^{(k)}\right)-A^{\prime}\left(s, u^{(0)}\right), u^{(k)}-u^{(0)}\right\rangle \\
& +\sum_{n=-\infty}^{+\infty} \varrho_{n}\left(g\left(s, u_{n}^{(k)}\right)-g\left(s, u_{n}^{(0)}\right)\right)\left(u^{(k)}-u^{(0)}\right) .
\end{aligned}
$$

Since $\left\langle A^{\prime}\left(s, u^{(k)}\right)-A^{\prime}\left(s, u^{(0)}\right), u^{(k)}-u^{(0)}\right\rangle \rightarrow 0$ as $k \rightarrow \infty$ and $\underline{v}>\tau>0, u^{(k)} \rightarrow u^{(0)}$ in $E$. So the proof is complete.

The following lemma provides the main mathematical result in the sequel. 
Lemma 3.1 Let $E \subset L^{0}\left(\Gamma_{S}\right)$ and $\mathcal{L}_{E}$ be a mapping from $L^{0}\left(\Gamma_{S}\right)$ onto $E$. If

$$
\mathcal{L}_{E}(x)=\arg \min _{y \in c}\|x-y\|
$$

for any $x \in L^{0}\left(\Gamma_{S}\right)$, then $\mathcal{L}_{E}$ is called the orthogonal projection from $L^{0}\left(\Gamma_{S}\right)$ onto E. Furthermore, we have the following properties:

(I) $\left\langle x-\mathcal{L}_{E} x, z-\mathcal{L}_{E} x\right\rangle \leq 0$;

(II) $\left\|\mathcal{L}_{E} x-\mathcal{L}_{E} y\right\|^{2} \leq\left\langle\mathcal{L}_{E} x-\mathcal{L}_{E} y, x-y\right\rangle$;

(III) $\left\|\mathcal{L}_{E} x-z\right\|^{2} \leq\|x-z\|^{2}+\left\|\mathcal{L}_{E} x-x\right\|^{2}$

for any $x, y \in L^{0}\left(\Gamma_{S}\right)$ and $z \in E$.

Our main result reads as follows.

Theorem 3.2 Let assumptions (H1)-(H3) hold. Then there exis's nique solution $(\mathfrak{X}, \mathfrak{Y}, \mathfrak{Z}, \mathfrak{K})$ for the NSD equation $(1)$.

Proof Existence. By Lemma 2.1, when $\alpha=0$, for $\forall \beta ., \varrho ., \lambda ., \varphi$., $\quad \in \operatorname{wr}_{G}(0, S), \xi \in L_{G}^{2}(\Gamma)$, (1) has a solution. Moreover, by Lemma 2.2, we can solv (2) succt ively for the case $\alpha \in$ $\left\lfloor 0, \delta_{0}\right],\left[\delta_{0}, 2 \delta_{0}\right], \ldots$ It turns out that, when $\alpha=1$, for $\forall \beta ., \varrho, \lambda, \gamma \psi \in M_{G}^{2}(0, S), \xi \in L_{G}^{2}(\Gamma)$, the solution of (1) exists, then we deduce that the solution of the NSD equation (1) exists.

Now, we prove the uniqueness.

Let $(u, \mathfrak{K})=(\mathfrak{X}, \mathfrak{Y}, \mathfrak{Z}, \mathfrak{K})$ and $\left(u^{\prime}, \mathfrak{K}^{\prime}\right)=\left(\mathfrak{X}^{\prime}, \mathfrak{Y},{ }^{\prime}, \mathfrak{K}^{\prime}\right)$ e two solutions of the NSD equation (1). We set

$$
\left(\hat{\mathfrak{X}}_{s}, \hat{\mathfrak{Y}}_{s}, \hat{\mathfrak{Z}}_{s}, \hat{\mathfrak{K}}_{s}\right):=\left(\mathfrak{X}_{s}-\mathfrak{X}^{\prime}-\mathfrak{Y}_{s}^{\prime}, \mathfrak{\nu}_{y}, \mathfrak{Z}_{s}^{\prime}, \mathfrak{K}_{s}-\mathfrak{K}_{s}^{\prime}\right) .
$$

From $(\mathrm{H} 1)-(\mathrm{H} 2)$, it is easy to see $\mathrm{t}$, at

$$
\left.\hat{\mathfrak{E}}\left[\sup _{0 \leq s \leq S}\left|\hat{\mathfrak{X}}_{s}\right|^{2}\right]+\hat{\mathfrak{E}} \underline{0 \leq s \leq S}\left|\hat{\mathfrak{y}}_{s}\right|^{2}\right]<\infty .
$$

In viey he property of the projection (see [30]), we infer that $\hat{u}=\mathcal{L}_{S_{i}}(\hat{u}-t \mathfrak{X} * \mathfrak{X} \hat{u})$ for any $s, F_{0}, \ldots$ we get from condition in (17) that

$$
{ }_{n} \leq \frac{2}{\rho\left(\mathfrak{X}^{*} \mathfrak{X}\right)} \mathfrak{Z}_{n} .
$$

follows that $I-\frac{\mu_{n}}{\mathfrak{Z}_{n}} \mathfrak{X} \mathfrak{X}$ is nonexpansive. Hence,

$$
\begin{aligned}
\left\|u_{n+1}-\hat{u}\right\|= & \left\|\mathcal{L}_{S_{i}}\left\{u_{n}-\mu_{n} \mathfrak{X}^{*} \mathfrak{X} v_{n}+\mathfrak{Z}_{n}\left(v_{n}-u_{n}\right)\right\}-\mathcal{L}_{S_{i}}\left\{\hat{u}-t \mathfrak{X}^{*} \mathfrak{X} \hat{u}\right\}\right\| \\
= & \| \mathcal{L}_{S_{i}}\left\{\left(1-\mathfrak{Z}_{n}\right) u_{n}+\mathfrak{Z}_{n}\left(I-\frac{\mu_{n}}{\mathfrak{Z}_{n}} \mathfrak{X}^{*} \mathfrak{X}\right) v_{n}\right\} \\
& -\mathcal{L}_{S_{i}}\left\{\left(1-\mathfrak{Z}_{n}\right) \hat{u}+\mathfrak{Z}_{n}\left(I-\frac{\mu_{n}}{\mathfrak{Z}_{n}} \mathfrak{X}^{*} \mathfrak{X}\right) \hat{u}\right\} \| \\
\leq & \left(1-\mathfrak{Z}_{n}\right)\left\|u_{n}-\hat{u}\right\|+\mathfrak{Z}_{n}\left\|\left(I-\frac{\mu_{n}}{\mathfrak{Z}_{n}} \mathfrak{X}^{*} \mathfrak{X}\right) v_{n}-\left(I-\frac{\mu_{n}}{\mathfrak{Z}_{n}} \mathfrak{X}^{*} \mathfrak{X}\right) \hat{u}\right\| \\
\leq & \left(1-\mathfrak{Z}_{n}\right)\left\|u_{n}-\hat{u}\right\|+\mathfrak{Z}_{n}\left\|v_{n}-\hat{u}\right\| .
\end{aligned}
$$


Since $\alpha \rightarrow 0$ as $n \rightarrow \infty$ and $\mathfrak{K}_{n} \in\left(0, \frac{2}{\rho\left(\mathfrak{X}^{*} \mathfrak{X}\right)}\right)$, it follows from (18) that

$$
\alpha \leq 1-\frac{\mathfrak{K}_{n} \rho\left(\mathfrak{X}^{*} \mathfrak{X}\right)}{2}
$$

as $n \rightarrow \infty$, that is,

$$
\frac{\mathfrak{K}_{n}}{1-\mathfrak{Y}_{n}} \in\left(0, \frac{\rho\left(\mathfrak{X}^{*} \mathfrak{X}\right)}{2}\right)
$$

We deduce from (18) that

$$
\begin{aligned}
\left\|v_{n}-\hat{u}\right\| & =\left\|\mathcal{L}_{S_{i}}\left\{\left(1-\mathfrak{Y}_{n}\right) u_{n}-\mathfrak{K}_{n} \mathfrak{X}^{*} \mathfrak{X} u_{n}\right\}-\mathcal{L}_{S_{i}}\left\{\hat{u}-t \mathfrak{X}^{*} \mathfrak{X} \hat{u}\right\}\right\| \\
& \leq\left(1-\mathfrak{Y}_{n}\right)\left(u_{n}-\frac{\mathfrak{K}_{n}}{1-\mathfrak{Y}_{n}} \mathfrak{X}^{*} \mathfrak{X} u_{n}\right)+\left\{\mathfrak{Y}_{n} \hat{u}+\left(1-\mathfrak{Y}_{n}\right)\left(\hat{u}-1-\mathfrak{X}^{*} \mathfrak{X} \hat{u}\right\}\right. \\
& \leq \|-\mathfrak{Y}_{n} \hat{u}+\left(1-\mathfrak{Y}_{n}\right)\left[u_{n}-\frac{\mathfrak{K}_{n}}{1-\mathfrak{Y}_{n}} \mathfrak{X}^{*} \mathfrak{X} u_{n}-\hat{u}+1\right.
\end{aligned}
$$

which is equivalent to

$$
\left\|v_{n}-\hat{u}\right\| \leq \mathfrak{Y}_{n}\|-\hat{u}\|+\left(1-\mathfrak{Y}_{n}\right)\left\|u_{n}-\hat{u}\right\|
$$

We obtain from (19)

$$
\begin{aligned}
\left\|u_{n}-\hat{u}\right\| & \leq\left(1-\mathfrak{Z}_{n}\right) \| u_{n}-\mathfrak{Z}_{n}\left(\mathfrak{Y}_{n},-\hat{u}\left\|+\left(1-\mathfrak{Y}_{n}\right)\right\| u_{n}-\hat{u} \|\right) \\
& \leq\left(1-\mathfrak{Z}_{n} \mathfrak{9} \mathfrak{J}_{n}\right)\left\|u_{n}-\hat{u}\right\|_{1} \mathfrak{Z}_{n} \mathfrak{Y}_{n}\|-\hat{u}\| \\
& \leq \max \{-\quad-\hat{u}\|, \downarrow-\hat{u}\|\} .
\end{aligned}
$$

So

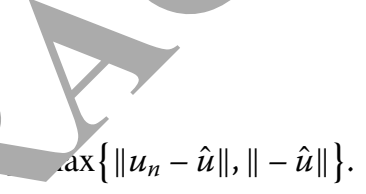

C sequently, $u_{n}$ is bounded, and so is $v_{n}$. Let $T=2 \mathcal{L}_{S_{i}}-I$. From Lemma 2.1, one can know that the projection operator $\mathcal{L}_{S_{i}}$ is monotone and nonexpansive, and $2 \mathcal{L}_{S_{i}}-I$ is nonex ansive.

So

$$
\begin{aligned}
u_{n+1} & =\frac{I+T}{2}\left[\left(1-\mathfrak{Z}_{n}\right) u_{n}+\mathfrak{Z}_{n}\left(1-\frac{\mu_{n}}{\mathfrak{Z}_{n}} \mathfrak{X}^{*} \mathfrak{X}\right) v_{n}\right] \\
& =\frac{I-\mathfrak{Z}_{n}}{2} u_{n}+\frac{\mathfrak{Z}_{n}}{2}\left(I-\frac{\mu_{n}}{\mathfrak{Z}_{n}} \mathfrak{X}^{*} \mathfrak{X}\right) v_{n}+\frac{T}{2}\left[\left(1-\mathfrak{Z}_{n}\right) u_{n}+\mathfrak{Z}_{n}\left(I-\frac{\mu_{n}}{\mathfrak{Z}_{n}} \mathfrak{X}^{*} \mathfrak{X}\right) v_{n}\right]
\end{aligned}
$$

which yields

$$
u_{n+1}=\frac{1-\mathfrak{Z}_{n}}{2} u_{n}+\frac{1+\mathfrak{Z}_{n}}{2} b_{n},
$$


where

$$
b_{n}=\frac{\mathfrak{Z}_{n}\left(I-\frac{\mu_{n}}{\mathfrak{Z}_{n}} \mathfrak{X}^{*} \mathfrak{X}\right) v_{n}+T\left[\left(1-\mathfrak{Z}_{n}\right) u_{n}+\mathfrak{Z}_{n}\left(I-\frac{\mu_{n}}{\mathfrak{Z}_{n}} \mathfrak{X}^{*} \mathfrak{X}\right) v_{n}\right]}{1+\mathfrak{Z}_{n}} .
$$

On the other hand, we have (see [31])

$$
\begin{aligned}
\left\|b_{n+1}-b_{n}\right\| \leq & \frac{\lambda_{n+1}}{1+\lambda_{n+1}}\left\|\left(I-\frac{\mu_{n+1}}{\lambda_{n+1}} \mathfrak{X}^{*} \mathfrak{X}\right) v_{n+1}-\left(I-\frac{\mu_{n}}{\mathfrak{Z}_{n}} \mathfrak{X}^{*} \mathfrak{X}\right) v_{n}\right\| \\
& +\left|\frac{\lambda_{n+1}}{1+\lambda_{n+1}}-\frac{\lambda_{n}}{1+\lambda_{n}}\right|\left\|\left(I-\frac{\mu_{n}}{\mathfrak{Z}_{n}} \mathfrak{X}^{*} \mathfrak{X}\right) v_{n}\right\| \\
& +\frac{T}{1+\lambda_{n+1}}\left\{\left(1-\lambda_{n+1}\right) u_{n+1}+\lambda_{n+1}\left(I-\frac{\mu_{n+1}}{\lambda_{n+1}} \mathfrak{X}^{*} \mathfrak{X}\right) v_{n+1}\right\} \\
& -\frac{T}{1+\lambda_{n+1}}\left\{\left[\left(1-\lambda_{n}\right) u_{n}+\lambda_{n}\left(I-\frac{\mu_{n}}{\lambda_{n}} \mathfrak{X}^{*} \mathfrak{X}\right) v_{n}\right]\right\} \\
& +\left|\frac{1}{1+\lambda_{n+1}}-\frac{1}{1+\lambda_{n}}\right|\left\|T\left[\left(1-\lambda_{n}\right) u_{n}+\lambda_{n}\left(1-\mu_{l}\right) v_{n}\right]\right\|
\end{aligned}
$$

For convenience, let $c_{n}=\left(I-\frac{\mu_{n}}{\lambda_{n}} \mathfrak{X}^{*} \mathfrak{X}\right) v_{n}$. Using Lemma 2.2, s, jlows that

$$
I-\frac{\mu_{n}}{\lambda_{n}} \mathfrak{X}^{*} \mathfrak{X}
$$

is nonexpansive and averaged.

Hence,

$$
\begin{aligned}
\left\|b_{n+1}-b_{n}\right\| \leq & \frac{\lambda_{n+1}}{1+}\left\|c_{n+1}-c_{n+1}\right\|+\frac{\lambda_{n+1}}{1+\lambda_{n+1}}-\frac{\lambda_{n}}{1+\lambda_{n}} \mid\left\|c_{n}\right\| \\
& \left.+\frac{1+\lambda_{n+1}}{1}\left(1-\lambda_{n+1}\right) u_{n+1}+\lambda_{n+1} c_{n+1}-\left[\left(1-\lambda_{n}\right) u_{n}+\lambda_{n} c_{n}\right]\right\} \\
& +\left|\frac{1}{1+\lambda_{n+1}}-\frac{1}{1+\lambda_{n}}\right|\left\|T\left[\left(1-\lambda_{n}\right) u_{n}+\lambda_{n} c_{n}\right]\right\| \\
\leq & \frac{\lambda_{n+1}}{1+\lambda_{n+1}}\left\|c_{n+1}-c_{n}\right\|+\left|\frac{\lambda_{n+1}}{1+\lambda_{n+1}}-\frac{\lambda_{n}}{1+\lambda_{n}}\right|\left\|c_{n}\right\| \\
& +\frac{1-\lambda_{n+1}}{1+\lambda_{n+1}}\left\|u_{n+1}-u_{n}\right\|+\frac{\lambda_{n+1}}{1+\lambda_{n+1}}\left\|c_{n+1}-c_{n}\right\|+\frac{\lambda_{n}-\lambda_{n+1}}{1+\lambda_{n+1}}\left\|u_{n}\right\| \\
& +\frac{\lambda_{n+1}-\lambda_{n}}{1+\lambda_{n+1}}\left\|c_{n}\right\|+\left|\frac{1}{1+\lambda_{n+1}}-\frac{1}{1+\lambda_{n}}\right|\left\|T\left[\left(1-\lambda_{n}\right) u_{n}+\lambda_{n} c_{n}\right]\right\|,
\end{aligned}
$$

which yields

$$
\begin{aligned}
\left\|c_{n+1}-c_{n}\right\| & =\left\|\left(I-\frac{\mu_{n+1}}{\lambda_{n+1}} \mathfrak{X}^{*} \mathfrak{X}\right) v_{n+1}-\left(I-\frac{\mu_{n}}{\lambda_{n}} \mathfrak{X}^{*} \mathfrak{X}\right) v_{n}\right\| \\
& \leq\left\|v_{n+1}-v_{n}\right\| \\
& =\left\|\mathcal{L}_{S_{i}}\left[\left(1-\alpha_{n+1}\right) u_{n+1}-\mathfrak{K}_{n} \mathfrak{X}^{*} \mathfrak{X} u_{n+1}\right]-\mathcal{L}_{S_{i}}\left[\left(1-\alpha_{n}\right) u_{n}-\mathfrak{K}_{n} \mathfrak{X}^{*} \mathfrak{X} u_{n}\right]\right\| \\
& \leq\left\|\left(I-\varrho_{n+1} \mathfrak{X}^{*} \mathfrak{X}\right) u_{n+1}-\left(I-\varrho_{n+1} \mathfrak{X}^{*} \mathfrak{X}\right) u_{n}+\left(\varrho_{n}-\varrho_{n+1}\right) \mathfrak{X}^{*} \mathfrak{X} u_{n}\right\|
\end{aligned}
$$




$$
\begin{aligned}
& +\alpha_{n+1}\left\|-u_{n+1}\right\|+\alpha_{n}\left\|u_{n}\right\| \\
\leq & \left\|u_{n+1}-u_{n}\right\|+\left|\varrho_{n}-\varrho_{n+1}\right|\left\|\mathfrak{X}^{*} \mathfrak{X} u_{n}\right\|+\alpha_{n+1}\left\|-u_{n+1}\right\|+\alpha_{n}\left\|u_{n}\right\| .
\end{aligned}
$$

So we infer that

$$
\begin{aligned}
\left\|b_{n+1}-b_{n}\right\| \leq & \left|\frac{\lambda_{n+1}}{1+\lambda_{n+1}}-\frac{\lambda_{n}}{1+\lambda_{n}}\right|\left\|c_{n}\right\|+\frac{\lambda_{n}-\lambda_{n+1}}{1+\lambda_{n+1}}\left\|u_{n}\right\|+\frac{\lambda_{n+1}-\lambda_{n}}{1+\lambda_{n+1}}\left\|c_{n}\right\| \\
& +\left\|u_{n+1}-u_{n}\right\|+\left|\frac{1}{1+\lambda_{n+1}}-\frac{1}{1+\lambda_{n}}\right|\left\|T\left[\left(1-\lambda_{n}\right) u_{n}+\lambda_{n} c_{n}\right]\right\| \\
& +\left|\varrho_{n}-\varrho_{n+1}\right|\left\|u_{n}\right\|+\alpha_{n+1}\left\|-u_{n+1}\right\|+\alpha_{n}\left\|u_{n}\right\| .
\end{aligned}
$$

By virtue of $\lim _{n \rightarrow \infty}\left(\lambda_{n+1}-\mathfrak{Z}_{n}\right)=0$ (see [28]), it follows that

$$
\lim _{n \rightarrow \infty}\left(\left|\frac{\lambda_{n+1}}{1+\lambda_{n+1}}-\frac{\lambda_{n}}{1+\lambda_{n}}\right|\right)=0
$$

Moreover, $\left\{u_{n}\right\}$ and $\left\{v_{n}\right\}$ are bounded, and so is $\left\{c_{n}\right\}$. T c are, $(20)$ reduces to

$$
\lim _{n \rightarrow \infty} \sup \left(\left\|b_{n+1}-b_{n}\right\|-\left\|u_{n+1}-u_{n}\right\|\right) \leq 0
$$

Applying (21) and Lemma 2.3, we oct

$$
\lim _{n \rightarrow \infty}\left\|b_{n}-u_{n}\right\|=0
$$

Combining (21) witl (22), we obtain

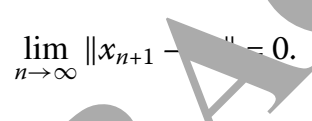

Apr. ng $\quad$ - to formula to $\hat{\mathfrak{X}}_{s} \hat{\mathfrak{Y}}_{s}$, then we obtain

$$
\begin{aligned}
& +\hat{\mathfrak{X}}_{S}\left[\Phi\left(\mathfrak{X}_{S}\right)-\Phi\left(\mathfrak{X}_{S}^{\prime}\right)\right]-\int_{0}^{S}\left[A\left(s, u_{s}\right)-A\left(s, u_{s}^{\prime}\right) u_{s}-u_{s}^{\prime}\right] d\langle B\rangle_{s} \\
& =\int_{0}^{S} \hat{\mathfrak{X}}_{s}\left[(-f)\left(s, u_{s}\right)-(-f)\left(s, u_{s}\right)\right]+\hat{\mathfrak{Y}}_{s}\left[b\left(s, u_{s}\right)-b\left(s, u_{s}^{\prime}\right)\right] d s+M_{S}
\end{aligned}
$$

from (23), where

$$
M_{s}=\int_{0}^{t}\left[\hat{\mathfrak{Y}}_{s}\left(\sigma\left(s, u_{s}\right)-\sigma\left(s, u_{s}^{\prime}\right)\right)+\hat{\mathfrak{X}}_{s} \hat{\mathfrak{Z}}_{s}\right] d \mathfrak{B}_{s}+\int_{0}^{t}\left(\hat{\mathfrak{X}}_{s}\right)^{+} d \mathfrak{K}_{s}+\int_{0}^{t}\left(\hat{\mathfrak{X}}_{s}\right)^{-} d \mathfrak{K}_{s}^{\prime}
$$

and

$$
N_{s}=\int_{0}^{t}\left(\hat{\mathfrak{X}}_{s}\right)^{+} d \mathfrak{K}_{s}^{\prime}+\int_{0}^{t}\left(\hat{\mathfrak{X}}_{s}\right)^{-} d \mathfrak{K}_{s}
$$


By Lemma 2.3 and (24), we know that both $M_{s}$ and $N_{s}$ are Schrödinger martingale. Moreover, we know that (see [32])

$$
\begin{aligned}
N_{S} & -(-C) \int_{0}^{S}\left|u_{s}-u_{s}^{\prime}\right|^{2} d\langle B\rangle_{s} \\
& \leq N_{S}+C\left|\hat{\mathfrak{X}}_{S}\right|^{2}+C \int_{0}^{S}\left|u_{s}-u_{s}^{\prime}\right|^{2} d\langle B\rangle_{s} \\
& \leq-\int_{0}^{S}\left|\hat{\mathfrak{X}}_{s}\right|^{2}+\left|\hat{\mathfrak{Y}}_{s}\right|^{2} d s+M_{S}
\end{aligned}
$$

from $(\mathrm{H} 3)$.

Taking the Schrödinger expectation on both sides of (25), together with Le. na 2......d the property of the Schrödinger expectation, we know that

$$
0 \leq-\underline{\sigma}^{2} \hat{\mathfrak{E}}\left[-C \int_{0}^{S}\left|u_{s}-u_{s}\right|^{2} d s\right] / \leq \hat{\mathfrak{E}}\left\{-\int_{0}^{S}\left[\left|\hat{\mathfrak{X}}_{s}\right|^{2}+\left|\hat{\mathfrak{Y}}_{s}\right|^{2}\right] d s\right\}=0
$$

which implies $u=u^{\prime}$ in the space of $M_{G}^{2}(0, S)$. It follow $m$ Ler.ma 2.2 that the NSD equation has a unique solution, then $K=K^{\prime}$. Thus (1) has? und que solution.

\section{Conclusions}

This paper was mainly devoted to the staciy of o kind of nonlinear Schrödinger differential equations. Under the integrable. in 'ary vaue condition, the existence and uniqueness of the solutions of this eqration w discussed by using new Riesz representations of linear maps and the Schre din $\varepsilon$ fixed point theorem.

\section{Acknowledgements}

The authors are thankful to the rable eviewers for their valuable suggestions and comments, which improved the paper. This paper was xitten during a short stay of the corresponding author at the Faculty of Science and Technology of Universiti Kebangsaâ in_as a visiting professor. She would also like to thank the school of Mathematics and their members for their warm ho spitgrity.

\section{Fundin}

This work uppund by the National Natural Science Foundation of China (No. 11526183) and the Shanxi Province Ed acation Sci "13th Five-Year" program (No. 16043).

Avaı. 'ity of data and materials

Not app, able.

\section{Co peting interests}

The authors declare that they have no competing interests.

\section{Authors' contributions}

All authors read and approved the final manuscript.

\section{Author details}

${ }^{1}$ College of Applied Mathematics, Shanxi University of Finance and Economics, Taiyuan, China. ${ }^{2}$ Department of Applied Mathematics, Yuncheng University, Yuncheng, China. ${ }^{3}$ Faculty of Science and Technology, Universiti Kebangsaan Malaysia, Bangi, Malaysia.

\section{Publisher's Note}

Springer Nature remains neutral with regard to jurisdictional claims in published maps and institutional affiliations. 


\section{References}

1. Caraballo, T., Robinson, J.C.: Stabilisation of linear PDEs by Stratonovich noise. Syst. Control Lett. 53(1), 41-50 (2004)

2. Laskin, N.: Fractional quantum mechanics and Lévy path integrals. Phys. Lett. A 268(4-6), 298-305 (2000)

3. Mohammed, S.A., Scheutzow, M.K.: Spatial estimates for stochastic flows in Euclidean space. Ann. Probab. 26(1), 56-77 (1998)

4. Rabinowitz, P.H.: On a class of nonlinear Schrödinger equations. Z. Angew. Math. Phys. 43(2), 270-291 (1992)

5. Kivshar, Y.S., Kevrekidis, G.P., Takeno, S.: Nonlinear localized modes in waveguide bends. Phys. Lett. A 307(5-6), 287-291 (2003)

6. Gross, E.P.: Structure of a quantized vortex in boson systems. Nuovo Cimento 20(10), 454-477 (1961)

7. Goubet, O., Hamraoui, E.: Blow-up of solutions to cubic nonlinear Schrödinger equations with defect: the radial case. Adv. Nonlinear Anal. 6(2), 183-197 (2017)

8. Bahrouni, A., Ounaies, H., Radulescu, V.: Infinitely many solutions for a class of sublinear Schrödinger equations with indefinite potentials. Proc. R. Soc. Edinb. A 145(3), 445-465 (2015)

9. Holzleitner, M., Kostenko, A., Teschl, G.: Dispersion estimates for spherical Schrödinger equations: the effect of boundary conditions. Opusc. Math. 36(6), 769-786 (2016)

10. Kristaly, A., Repovs, D.: On the Schrödinger-Maxwell system involving sublinear terms. Nonlinear Anal., Re World Appl. 13(1), 213-223 (2012)

11. Repovs, D., Ščepin, E.: A proof of the Hilbert-Smith conjecture for actions by Lipschitz maps. Matb 361-364 (1997)

12. Zhang, X., Chen, P.: Fractional evolution equation nonlocal problems with noncompact sem roups. Opu Math 36(1), 123-137 (2016)

13. Dorogovtsev, A.Ya., Le Vin'Tkhuan: Correlation functions of Gaussian Markov stationary and per crocesses in a Hilbert space. In: Selected Problems in the Current Theory of Random Processes, p $\quad 65$. Akad. a ak Ukrain. SSR, Inst. Mat., Kiev (1988) (Russian)

14. Swift, R.J.: Almost periodic harmonizable processes. Georgian Math. J. 3(3), 275-, (1

15. Tudor, C.: Almost periodic solutions of affine stochastic evolution equations. Stoch. ch. Rep. 38(4), 251-266 (1992)

16. Acquistapace, P., Terreni, B.: A unified approach to abstract linear nonau nomous pai Jíc equations. Rend. Semin. Mat. Univ. Padova 78, 47-107 (1987)

17. Karatzas, I., Shreve, S.E.: Brownian Motion and Stochastic Calculus, 2nd ed Gragy re Texts in Mathematics, vol. 113 Springer, New York (1991)

18. Lunardi, A.: Analytic Semigroups and Optimal Regularity in holic Proble is. Progress in Nonlinear Differential Equations and Their Applications, vol. 16. Birkhäuser, Ba el (199.

19. Sussmann, H.J.: On the gap between deterministic ana chastic dinary differential equations. Ann. Probab. 6(1), 19-41 (1978)

20. Dorogovtsev, A.Ya., Ortega, O.A.: On the existe ce of periodic ations of a stochastic equation in a Hilbert space. Visnik Kiiv. Univ. Ser. Mat. Mekh. 30, 21-30, 11. 98 .

21. Cyganowsky, S., Kloeden, P., Ombach, J. Tum Elo ntary robability to Stochastic Differential Equations with MAPLE. Springer, Berlin (2002)

22. Friedman, A.: Stochastic Differenti- Equ ns and Applications, vol. 1. Probability and Mathematical Statistics, vol. 28. Academic Press, New York (1975)

23. McKean, H.P. Jr:: Stochastic I itegrals. Probabily and Mathematical Statistics, vol. 5. Academic Press, New York (1969)

24. Ichikawa, A., Pritchard, A.J. xistence, uniqueness, and stability of nonlinear evolution equations. J. Math. Anal. Appl. 68(2), 454-476 (1979)

25. Kawabata, S., Yamada, T.: O method for stochastic differential equations. In: Séminaire de Probabilités, XXV. Lecture Notes in $\mathrm{h}$ vol. 148\%, pp. 121-137. Springer, Berlin (1991)

26. Arnold, L., Tudor, C Ju wand almost periodic solutions of almost periodic affine stochastic differential equations. Stoch. St d/. Rel, 64(3-4), 177-193 (1998)

27. Berglu 4 , Dissipa ive weakly almost periodic functions. Mat.-Fys. Medd. Danske Vid. Selsk. 42(3), 77-80 (1989)

28. Kay T:: A nost per, odic weakly stationary processes. In: Statistics and Probability: Essays in Honor of C. R. Rao, n. 38 . O.wu. -Holland, Amsterdam (1982)

20 Huang, J. "antive tracking control of high-order non-holonomic mobile robot systems. IET Control Theory Appl. (6), 681-6y, (2009)

30. $H$. Liens entre équations différentielles stochastiques et ordinaires. Ann. Inst. Henri Poincaré B, Calc. Probab. Sta $;(2), 99-125(1977)$

31. Yang, W., Duan, J., Hu, W., Zhang, J.: New Riesz representations of linear maps associated with certain boundary value problems and their applications. Bound. Value Probl. 2017, 165 (2017)

Wang, J., Huang, B., Yamini, N.: An augmented Riesz decomposition method for sharp estimates of certain boundary value problem. Bound. Value Probl. 2016, 156 (2016) 